Colorimetric Estimation of Phosphorus

To determine the rate of colour formation and the optimum time for taking readings in the Fiske and Subbarow method of estimating phosphorus microchemically, we have used a simple type of photoelectric colorimeter (to be described in detail elsewhere) which allows the chemical reaction to be followed continuously until a state of equilibrium is reached. The method has been developed for the purpose of obtaining more accurate figures for the phosphorus content of the whole blood and its separate fractions (plasma, serum and red cells) than those hitherto obtained by other (visual) methods.

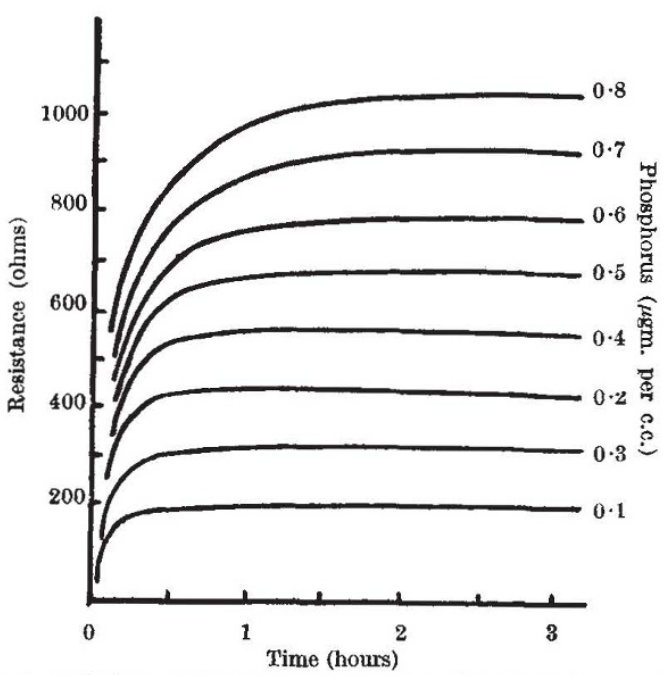

Fra. 1. Relation of colour intensity to time. Curves for the smallest amounts of phosphorus measured have been omitted ; in the interest of clearness, the steep ascents in the earlier stages of colour formation
have also been omitted.

Our apparatus enables us to detect $10^{-9} \mathrm{gm}$. of phosphorus per c.c. of solution, and to measure accurately changes of less than $10^{-7} \mathrm{gm}$. The reagent used, which differs slightly from that given by Fiske and Subbarow, consists of $0.1 \mathrm{gm}$. of $1: 2: 4$ aminonaphthol-sulphonic acid, $5.48 \mathrm{gm}$. of sodium metabisulphite, $1 \cdot 2 \mathrm{gm}$. of crystalline sodium sulphite dissolved in distilled water to make 50 c.c. of the solution. When the component substances are in these proportions, the acid remains in solution unless the temperature changes markedly. A beam of approximately monochromatic light which, passing through a liquid filter containing the solution to be examined, falls on to a photo-electric cell and so gives rise to a photo-electric current, is balanced against a current generated by a beam from the same source, which has, however, passed through a glass vessel containing only distilled water. After the addition of 2 c.c. of an ammonium molybdate and sulphuric acid solution and then of 1 c.c. of the modified Fiske and Subbarow reagent to the test solution, the characteristic blue colour that forms may be followed by means of a variable resistance which is manipulated so that the spot of light of a mirror galvanometer (of low resistance) remains undeflected.

By measuring solutions containing known amounts of phosphorus (from 0.03 to I microgram per c.c.) we calibrated the instrument to express intensity of colour in terms of the balancing resistance; it was found that, roughly, a difference of 0.05 microgram of phosphorus per c.c. produced a colour which caused a change of resistance of $50 \mathrm{ohms}$, a difference of $1 \mathrm{ohm}$ producing an easily perceptible deflection even with an ordinary laboratory galvanometer. The calibration curve is very nearly linear. When the intensity of colour, expressed in ohms, is plotted against time, curves of the type shown in Fig. 1 are obtained.

These curves show that the colour begins to form immediately after the reagents have been added and increases rapidly in intensity until a comparatively steady state is reached : actually, the colour continues to deepen for many hours. When the quantity of phosphorus present is very small, this approxi mately steady value is attained in about 20 minutes, but when the amounts are of the order of $0.5 \mu \mathrm{gm}$. per c.c., the colour should not be read until two hours have elapsed since the reagents were added. The curves shown in Fig. I are drawn from readings taken at intervals of $2 \frac{1}{2}$ minutes in the early stages, then 5 minutes, later 15 minutes and hourly. It also follows from these curves that, when the colours are read in the ordinary ocular colorimeters, the varying rates of development of colour in the standard and the unknown will escape notice owing to the relative insensitiveness of the method.

A merit of the present apparatus is that once the calibration curve for standard amounts of phos. phorus has been determined, it is no longer necessary to prepare a standard for comparison with the unknown solution, since the liquid filter used to obtain an electrical balance need contain only dis. tilled water. The advantage of being able to measure accurately the successive changes of colour in the solution is obvious.

The same method is now being applied to other standard colorimetric tests, and it appears probable that the interval of time within which the respective colours are to be read will be found, as in the present case, to be greater than that usually allowed, since more accurate results will be obtained when the measurements are made on the nearly horizontal branches of the resistance curves. In some tests, such as that for cholesterol, it will be possible to determine the optimum time for reading the colour before it begins to fade.

Under the auspices of the Cancer Research Com. mittee of the University of Sydney, we are using the above method to measure the amounts of certain constituent substances in pathological human blood.
New Medical School,
University,
HenRy L. Brose.
ERNEST B. Jones.

Sydney.

\section{Surface Properties of Non-Aqueous Solutions}

A close study of the surface properties of capillary. active solutions of compounds forming homologous series has shown that the surface activity increases regularly with the molecular weight (Traube's rule). Only aqueous solutions have, however, been thoroughly investigated, and little is known of non. aqueous systems. Since organic solvents have much lower surface tensions than water, the changes pro. duced by solutes will doubtless be much smaller, but the following results show that in certain solvents interesting effects of a similar nature can be observed.

I have determined the surface tension of solutions of different normal alcohols in aniline by means of a Traube stalagmometer. Aniline was chosen as solvent because of its comparatively high surface tension $\left(\varsigma_{80}{ }^{\circ}=42.9\right.$ dynes $/ \mathrm{cm}$.). Although the lowering of the surface tension $\left(\Delta_{\zeta}\right)$ is much smaller than in aqueous solutions, it was clearly established that, as 